\title{
A comprehensive genetic map of sugarcane that provides enhanced map coverage and integrates high-throughput Diversity Array Technology (DArT) markers
}

Karen S Aitken ${ }^{1 *}$, Meredith D McNeil1 ${ }^{1}$, Scott Hermann², Peter C Bundock ${ }^{3}$, Andrzej Kilian ${ }^{4}$, Katarzyna Heller-Uszynska ${ }^{4}$, Robert J Henry ${ }^{5}$ and Jingchuan $\mathrm{Li}^{1}$

\begin{abstract}
Background: Sugarcane genetic mapping has lagged behind other crops due to its complex autopolyploid genome structure. Modern sugarcane cultivars have from 110-120 chromosomes and are in general interspecific hybrids between two species with different basic chromosome numbers: Saccharum officinarum $(2 n=80)$ with a basic chromosome number of 10 and S. spontaneum $(2 n=40-128)$ with a basic chromosome number of 8 . The first maps that were constructed utilised the single dose (SD) markers generated using RFLP, more recent maps generated using AFLP and SSRs provided at most 60\% genome coverage. Diversity Array Technology (DArT) markers are high throughput allowing greater numbers of markers to be generated.

Results: Progeny from a cross between a sugarcane variety Q165 and a S. officinarum accession IJ76-514 were used to generate 2467 SD markers. A genetic map of Q165 was generated containing 2267 markers, These markers formed 160 linkage groups (LGs) of which 147 could be placed using allelic information into the eight basic homology groups (HGs) of sugarcane. The HGs contained from 13 to 23 LGs and from 204 to 475 markers with a total map length of $9774.4 \mathrm{cM}$ and an average density of one marker every $4.3 \mathrm{cM}$. Each homology group contained on average 280 markers of which $43 \%$ were DArT markers 31\% AFLP, 16\% SSRs and 6\% SNP markers. The multi-allelic SSR and SNP markers were used to place the LGs into HGs.

Conclusions: The DArT array has allowed us to generate and map a larger number of markers than ever before and consequently to map a larger portion of the sugarcane genome. This larger number of markers has enabled $92 \%$ of the LGs to be placed into the $8 \mathrm{HGs}$ that represent the basic chromosome number of the ancestral species, S. spontaneum. There were two HGs (HG2 and 8) that contained larger numbers of LGs verifying the alignment of two sets of $S$. officinarum chromosomes with one set of $\mathrm{S}$. spontaneum chromosomes and explaining the difference in basic chromosome number between the two ancestral species. There was also evidence of more complex structural differences between the two ancestral species.
\end{abstract}

Keywords: Saccharum, Polyploid, Genetic mapping, SNP markers, DArT sugarcane array

\footnotetext{
* Correspondence: Karen.aitken@csiro.au

${ }^{1}$ CSIRO Plant Industry, Queensland Bioscience Precinct, 306 Carmody Rd,

St Lucia, QLD 4067, Australia

Full list of author information is available at the end of the article
} 


\section{Background}

Sugarcane is widely cultivated in tropical and subtropical regions and is primarily grown for sugar production accounting for about $75 \%$ of the world's sucrose supply. It is clonally propagated and has a very high photosynthetic efficiency which makes it very attractive as a source of biomass [1]. Recently it has become more important as a biofuel crop for the production of ethanol [2]. It is a perennial grass, a member of the Poaceae family and Andropogoneae tribe, which includes maize and sorghum. Cultivated sugarcane is derived from inter-specific hybridisation between, in the main, two polyploid species S. officinarum L. and S. spontaneum L which have different basic chromosome numbers. The resultant cultivars are complex aneu-polyploids with chromosome numbers of $2 n=100-120$. In the production of modern cultivars derived from the initial hybrid, the $2 \mathrm{n}$ transmission that occurs from $S$. officinarum in the primary cross has been used to advantage. Backcrossing to S. officinarum or other cultivars recovers the high sugar phenotype inherited from $S$. officinarum while retaining the disease resistance and good ratooning of the wild S. spontaneum parent. The preferential outbreeding, highly heterozygous and predominantly autopolyploid genetics of sugarcane are all factors that have hindered the development of a comprehensive genetic map.

Classic cytological studies using in situ hybridization have determined that $S$. officinarum is an octaploid $(2 \mathrm{n}=8 \mathrm{x}=80)$ with a basic chromosome number of 10 , the same as its closest known diploid relative sorghum [3]. S. spontaneum has varying ploidy levels $(2 n=40-180)$ but has a basic chromosome number of $\mathrm{x}=8$ [3]. Genome in situ hybridisation has determined that commercial cultivars contain approximately $80 \% \mathrm{~S}$. officinarum chromosomes, $10-15 \% S$. spontaneum chromosomes and approximately $10 \%$ recombinant chromosomes [4,5]. Q165 is an Australian cultivar no longer grown commercially. It has 110 chromosomes of which $82-83$ (75\%) are inherited from S. officinarum, 15-17 (15\%) are inherited from S. spontaneum and 11-12 (11\%) are recombinant chromosomes [3]. These are similar in the proportions found in most varieties studied to date [5].

Sugarcane has a large genome $(10 \mathrm{~Gb})[6]$ and in the absence of a genome sequence, a high-density genetic map is a valuable tool to understand the genetic and genomic organisation of this complex polyploid crop. Its autopolyploid nature with mostly random pairing plus high inbreeding depression has limited the production of more common experimental mapping populations such as double haploids or recombinant inbred lines. The complication of the coexistence of single dose (SD) and multi dose alleles and irregular chromosome numbers in the various homo(eo)logy classes due to aneuploidy has restricted genetic mapping. With the realisation that
SD markers can be used to generate genetic maps in polyploids [7] genetic maps were developed in sugarcane using a population of full-sib $\left(\mathrm{F}_{1}\right)$ individuals (pseudo-test cross strategy) [8] with SD markers segregating 1:1 or by using a population created by selfing an individual and mapping SD markers segregating 3:1. Initially mapping was carried out in the ancestral species S. spontaneum [9-11] and S. officinarum $[12,13]$. More recently cultivars of hybrid origin have been mapped [14-20]. All of the genetic maps that have been generated for sugarcane to date have had low genome coverage and limited information on genome organisation. One of the main reasons for this has been the limited number of markers mapped. In most cases fewer than 1000 have been mapped due to the complexity of marker generation in such a complex polyploidy and the high cost of generation of large numbers of mappable markers. Many markers of different dosage are generated in sugarcane but segregation of markers of greater than double dose in the 200-300 size populations means that accuracy of marker placement is low [7].

The development of Diversity Array Technology (DArT) [21] for sugarcane has combined the low-cost highthroughput properties of the DNA microarray platform with the ability to identify various types of DNA polymorphisms [22].

This paper reports the generation of a comprehensive sugarcane genetic map of Q165, an Australian sugarcane incorporating 2267 markers generated from DArT, amplified fragment length polymorphism (AFLP), simple sequence repeats (SSR), single nucleotide polymorphism (SNP), restriction fragment length polymorphism (RFLP) and random amplified polymorphism (RAPD) markers. This large number of markers allowed the majority of the LGs to be placed into the 8 homology groups which is consistent with the basic chromosome number of the ancestral species S. spontaneum and the lowest basic chromosome number identified in the Saccharum genus.

\section{Results}

\section{Generation of mapping data}

To generate the maximum number of polymorphic markers using the sugarcane DArT array, the DNA from each of the $F_{1}$ progeny was digested with several different restriction enzyme combinations. DNA from each combination was then screened separately across the sugarcane DArT array [22]. Using these separately screened enzyme combinations, a higher number of polymorphic markers was generated. In total 1555 markers were scored as present/absent of which 726 were generated from a PstI/BstNI digest, 640 from a PstI/HpaII digest and 189 from a PstI/TaqI digest. Of the 1555 DArT markers generated 263 were identified by more than one enzyme digest and had the same segregation 
pattern. The remaining 1246 unique markers included 1018 (82\%) which were SD and used to generate the linkage map. PstI/BstNI generated 580 (80\%) SD markers, PstI/HpaII generated 528 (83\%) and PstI/TaqI generated 163 (87\%).

In total 3475 markers were scored. Of these, 598 were SSR markers of which 473 were SD, 1062 AFLP markers of which 770 were SD, 184 SNP markers of which 153 were SD, 43 RAPD markers of which 26 were SD; and 33 were RFLP markers of which 27 were SD (Table 1; Additional file 1). The majority of the segregating markers inherited from Q165 were SD, and segregating 1:1 (present:absent) in the population (Tables 1 and 2).

\section{Genetic map construction}

The genetic map was constructed using 227 progeny generated from a cross between Q165 an old Australian cultivar and IJ76-514 a S. officinarum accession using the pseudo testcross approach. Of the 3475 markers scored that were present in Q165 and absent in IJ76514, 2467 (78\%) were SD in Q165. The majority of SD markers were DArT, AFLP and SSR markers although 184 EST SNP markers were also scored in this population (Table 2). Of the 2467 SD markers used for mapping, 967 were present in and used to generate the map reported in [15]. Although the markers were selected as SD markers segregating 1:1 in the progeny a chi square value was used that allowed inclusion of SD markers with moderate segregation distortion (skewed SD markers) but excluded likely double dose markers as in [23], as it is well known that segregation distortion is common in plants. The percentage of skewed markers varied from homology group (HG) to HG with $18 \%$ of markers skewed in HG7 and $37.5 \%$ of markers skewed in HG5 (Figure 1). Initially for map construction, only the markers that were present in greater than 188 progeny

Table 1 Total number of single dose (SD), double dose (DD), triple dose (TD) and bi-parental SD markers (3:1 markers) scored in the parents of the mapping population

\begin{tabular}{lll}
\hline & $\begin{array}{l}\text { sugarcane cultivar (hybrid) } \\
\text { Q165 }\end{array}$ & $\begin{array}{l}\text { S. officinarum } \\
\text { IJ76-514 }\end{array}$ \\
\hline Chromosome no. & 110 & 80 \\
Number of markers & 3182 & 1168 \\
Number of SD markers & 2467 & 529 \\
Number of DD markers & 486 & 444 \\
Number of TD markers & 17 & 1 \\
Number of 3:1 markers & 256 & \\
Total number of LG & 160 & $66^{1}$ \\
$\begin{array}{l}\text { No. of SD markers } \\
\text { in map }\end{array}$ & 2267 & - \\
\hline
\end{tabular}

${ }^{1}$ The addition of DArT markers did not provide the same magnitude of improvement to the IJ76-514 linkage map and the data is not shown
Table 2 Number of markers generated with the different marker systems and number of single dose (SD) markers (as a percentage of the total number)

\begin{tabular}{lll}
\hline Markers & Q165 & SD (\%) \\
\hline DArT & 1246 & $1018(82 \%)$ \\
AFLP & 1062 & $770(73 \%)$ \\
SSR & 598 & $473(79 \%)$ \\
SNP & 184 & $153(83 \%)$ \\
RAPD & 43 & $26(60 \%)$ \\
RFLP & 33 & $27(82 \%)$ \\
Total & 3182 & 2467 \\
\hline
\end{tabular}

were used to generate a framework map. A number of the DArT markers (335 of the 1018 SD markers) were only scored in 94 individuals, and these markers were added to the map after initial map construction using markers scored across the whole population. The 2467 SD markers formed 160 linkage groups containing 2267 markers, leaving 200 (8\%) unlinked markers (Table 3, Figure 2).

\section{Assembly of Saccharum homo(eo)logous groups (HGs)}

As sugarcane is an autopolyploid it contains many copies of each chromosome. In order to determine the genome structure of Q165 the linkage groups (LGs) were placed into HGs using a number of different strategies. Firstly using the multi-allelic loci detected by single SSR, RFLP or SNP markers, secondly using sequence information from these markers and thirdly using repulsion phase analysis. The majority of the multi-allelic markers were from SSRs but a major problem encountered with the SSR markers was that only $47 \%$ of the 116 SSRs derived from sugarcane that were screened across the map, were specific to one homology group. Thirty-five of the SSRs had only one SD segregating marker and 26 (22\%) mapped to more than one homology group. For the 43 SSRs derived from sorghum that were mapped, only $8(19 \%)$ were specific to one homology group and the majority (27 markers, 63\%) only generated one SD marker. Nevertheless, of the 160 linkage groups formed, 147 (92\%) could be placed into HGs. These HGs contained from 13 to 23 LGs, with the LGs varying in length from $1.3 \mathrm{cM}$ (consisting of two markers), to 232.7 cM (consisting of 81 markers) (Table 3, Figure 2). Coverage within the homology groups varied from HG2 with a total map length of $1818 \mathrm{cM}$ to HG6 with a total map length of $678.3 \mathrm{cM}$ (Table 3). HG 2 also had the most markers mapped with 475 markers compared to HG7 which contained the least number of markers at 204 (Table 3).

\section{Chromosome pairing within HGs}

To determine whether there is preferential pairing behaviour within Q165 we examined linkage in repulsion 

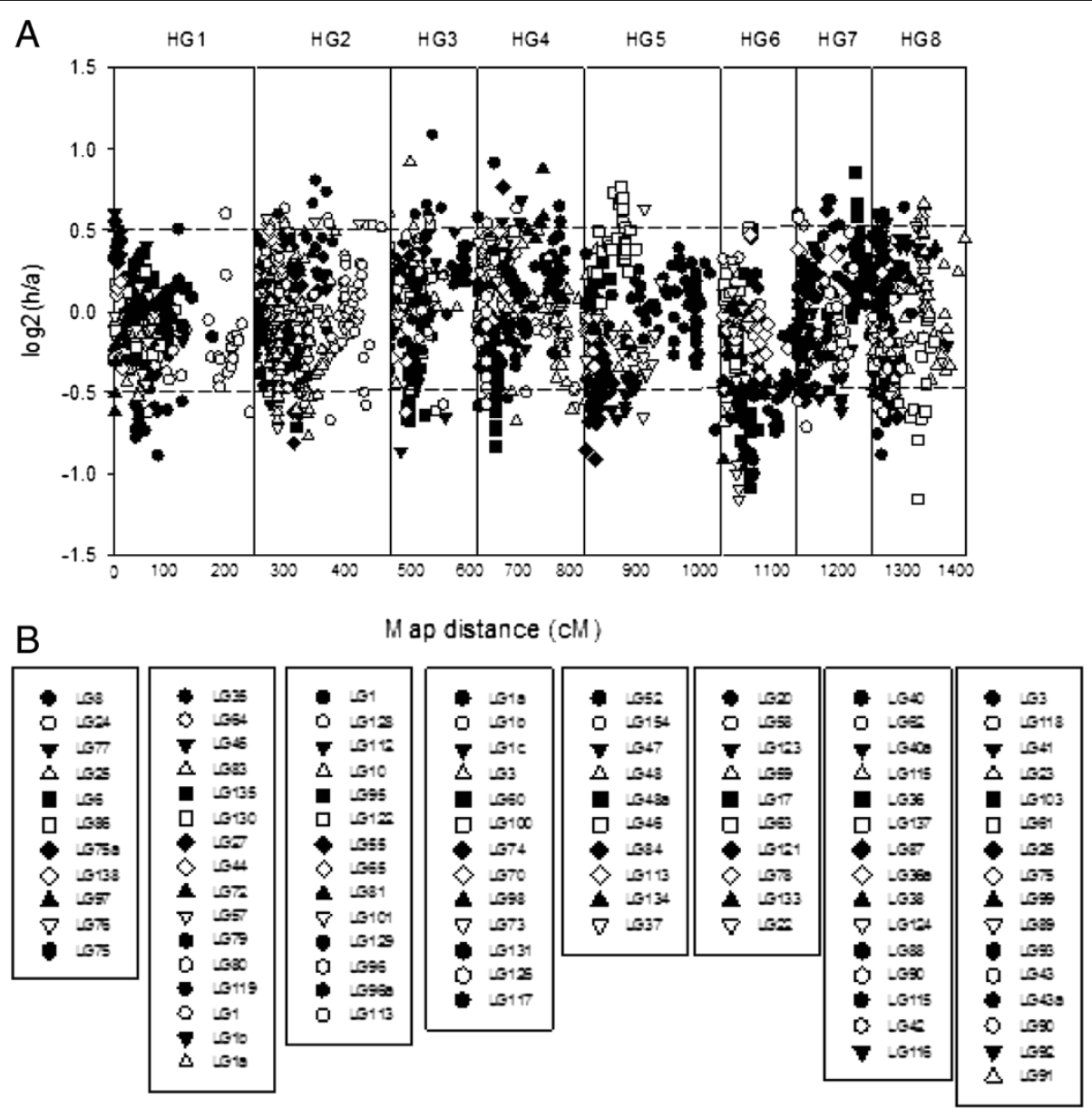

Figure 1 Scatter plot representing the distribution of marker segregation distortion within each LG within the HGs with each dot representing one molecular marker. The vertical solid lines distinguish the $8 \mathrm{HGs}$ which represent the 8 basic chromosomes, along the total map distance ( $x$ axis). The $y$ axis is the log2 value of the ratio of the number of individuals carrying the marker compared to the number of individuals not carrying the marker. Markers outside the two horizontal dotted lines are significantly skewed as calculated by the Chi-square test at $p=0.05$.

Table 3 The number of each type of marker mapped within each homology group (HG) of Q165 and the number of linkage groups (LGs) formed within each (HG)

\begin{tabular}{lllllllllll}
\hline HG & No. LG & No. AFLP & No. DART & No. SSR & No. SNP & No. RFLP & No. RAPD & $\begin{array}{l}\text { Total number } \\
\text { of markers mapped }\end{array}$ & $\begin{array}{l}\text { Length of in cM } \\
\text { HG Marker density (cM) }\end{array}$ \\
\hline $\mathbf{1}$ & 17 & 82 & 125 & 60 & 13 & 1 & 1 & 282 & 1253.4 & 4.4 \\
$\mathbf{2}$ & 23 & 139 & 198 & 105 & 25 & 0 & 8 & 475 & 1818.0 & 3.8 \\
$\mathbf{3}$ & 20 & 77 & 75 & 52 & 11 & 2 & 1 & 218 & 1243.6 & 5.7 \\
$\mathbf{4}$ & 18 & 108 & 125 & 50 & 13 & 5 & 4 & 305 & 1210.7 & 3.9 \\
$\mathbf{5}$ & 13 & 71 & 94 & 32 & 24 & 1 & 0 & 222 & 846.6 & 3.8 \\
$\mathbf{6}$ & 18 & 69 & 111 & 34 & 13 & 2 & 3 & 232 & 678.3 & 2.9 \\
$\mathbf{7}$ & 16 & 62 & 90 & 27 & 12 & 8 & 5 & 204 & 1020.5 & 5.0 \\
$\mathbf{8}$ & 22 & 98 & 132 & 49 & 15 & 0 & 2 & 295 & 1319.2 & 4.4 \\
Unassigned & 13 & 19 & 10 & 3 & 0 & 0 & 2 & 34 & 384.1 & 10.9 \\
Total & 160 & 721 & 976 & 418 & 124 & 19 & 25 & 2267 & 9774.4 & 4.3
\end{tabular}




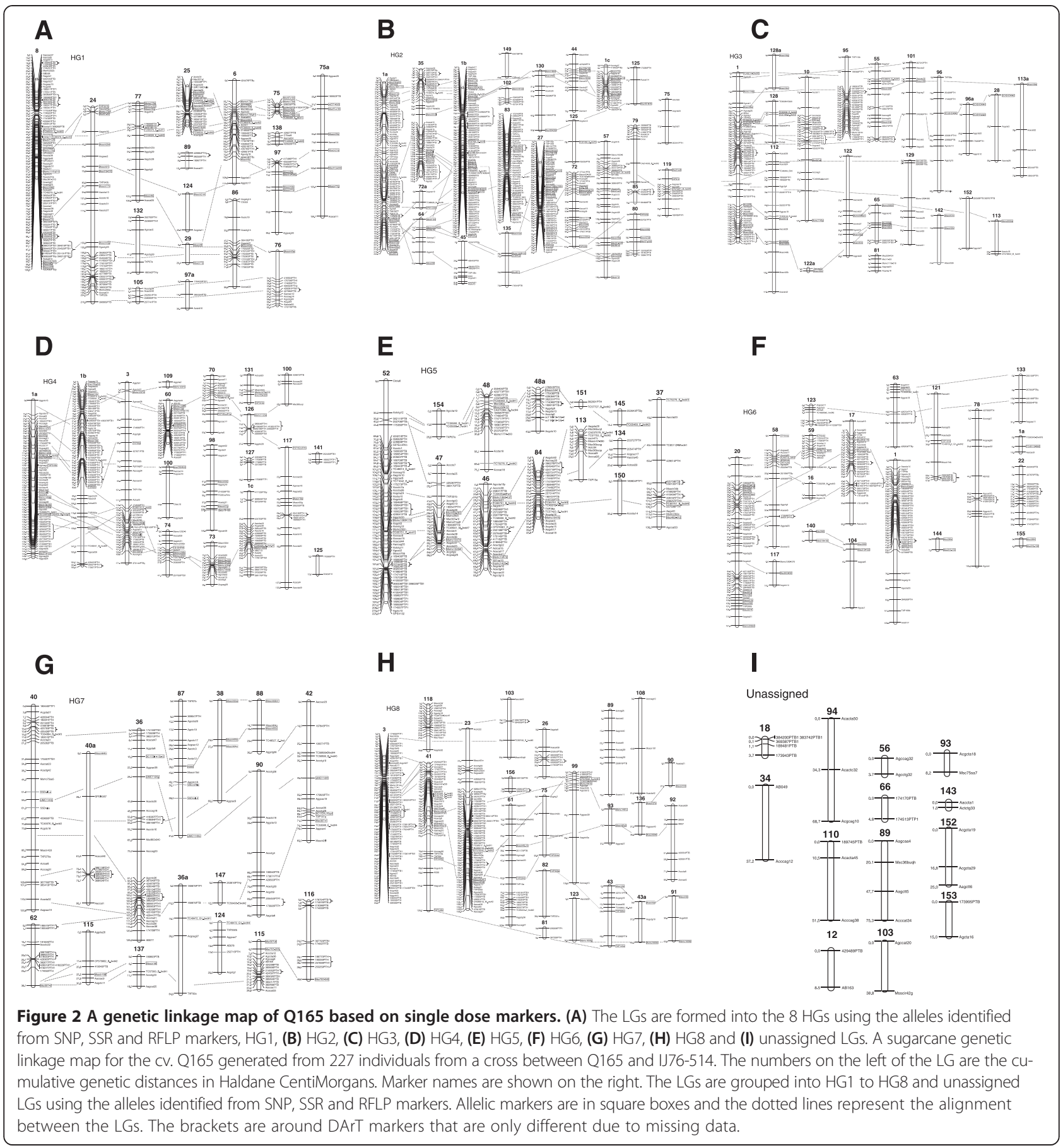

of each marker with all other markers in the map. A total of 30 LGs were linked in repulsion with other LGs. The amount of repulsion detected varied between HGs, with HG6 having 7 LGs (33\%) linked in repulsion compared to HG3 where no repulsion was detected (Table 4). In the HGs where repulsion was detected, the number of LGs involved varied from 2 to 7 . Out of the 30 LGs linked in repulsion, 6 pairs of LGs were only found in repulsion to each other. The other 18 LGs were in repulsion with more than one other LG. This indicated that in most cases there was preference in pairing between a small number of LGs. A difference ratio was calculated to compare how the genetic distances of the LGs within a HG varied between HGs [24]. One high coverage LG was selected per HG to compare to all other LGs within the same HG (Table 5). The average difference ratio varied from 0.13 for HG3 to 0.86 for HG6. 


\section{Intrachromosomal rearrangements within the homology groups \\ HG1}

HG1 contained 17 LGs which were grouped using 14 multi-allelic SSRs and 4 multi- allelic SNP markers. All of these multi-allelic markers were only mapped in HG1; there was only one allele from an SSR that mapped to another HG (Figure 2A). In general the LGs within the HG were syntenic to each other with the order of the markers highly conserved. Only HG1LG97 appeared to have a change in marker order at the end of the LG compared to other LGs within the HG with markers between M77i and M8a inverted.

\section{HG2}

This was one of the largest HGs with a total of 475 markers and the longest total length of $1818 \mathrm{cM}$ (Table 3 Figure 2B). There were 30 multi-allelic markers with a range of alleles from 2 to 9 in this HG. Combined with the sequence information from markers with known sequence this produced a high coverage HG. The order of markers for the lower coverage linkage groups was fairly consistent with minor order changes probably as a result of missing data. There were five LGs (HG2LG1a, HG2LG35, HG2LG1b, HG2LG83, HG2LG27) with extensive rearrangements which appear to be recombinant LGs. These chromosomes are on average $52 \%$ longer than the rest of the LGs within this HG. This HG group contains the largest number of LGs and from the SSR markers there are two distinct sets of smaller LGs that align to these larger LGs. One set of LGs containing SSRs Msscir 17, Msmc1604, Msscir 44, Msmc 1825 and Msscir 46 and the other set of LGs containing Msscir28, Msmc336, Msscir 12, Msc851. This indicates that this HG is composed of S. spontaneum chromosomes aligned to two sets of $S$. officinarum chromosomes in agreement with the basic chromosome number of these progenitor species.

Table 4 Repulsion analysis between LGs within HGs

\begin{tabular}{|c|c|c|c|c|c|}
\hline HG & Number of LGs in repulsion & $\begin{array}{l}\text { Number of LGs with } \\
\text { no repulsion detected }\end{array}$ & $\begin{array}{l}\text { Percentage of } \\
\text { LGs with repulsion }\end{array}$ & LGs in repulsion & LOD score of linkage \\
\hline \multirow[t]{2}{*}{1} & 3 & 14 & $18 \%$ & HG1LG8-HG1LG25 & 7 \\
\hline & & & & HG1LG8-HG1LG6 & 7 \\
\hline \multirow[t]{5}{*}{2} & 5 & 18 & $22 \%$ & HG2LG1a-HG2LG27 & 7 \\
\hline & & & & HG2LG1b-HG2LG1c & 8 \\
\hline & & & & HG2LG1a-HG2LG35 & 7 \\
\hline & & & & HG2LG1C-HG2LG35 & 8 \\
\hline & & & & HG2LG72a-HG2LG72 & 5 \\
\hline 3 & 0 & 20 & 0 & & \\
\hline 4 & 2 & 16 & $11 \%$ & HG4LG1c-HG4LG1a & 6 \\
\hline \multirow[t]{4}{*}{5} & 5 & 8 & $38 \%$ & HG5LG47-HG5LG46 & 12 \\
\hline & & & & HG5LG52-HG5LG46 & 7 \\
\hline & & & & HG5LG48-HG5LG48a & 10 \\
\hline & & & & HG5LG52-HG5LG47 & 7 \\
\hline \multirow[t]{7}{*}{6} & 7 & 11 & $33 \%$ & HG6LG20-HG6LG58 & 5 \\
\hline & & & & HG6LG20-HG6LG1 & 5 \\
\hline & & & & HG6LG20-HG6LG17 & 5 \\
\hline & & & & HG6LG20-HG6LG59 & 5 \\
\hline & & & & HG6LG58-HG6LG59 & 7 \\
\hline & & & & HG6LG17-HG6LG59 & 7 \\
\hline & & & & HG6LG16-HG6LG1b & 6 \\
\hline \multirow[t]{3}{*}{7} & 4 & 12 & $25 \%$ & HG7LG42-HG7LG36 & 6 \\
\hline & & & & HG7LG36-HG7LG36a & 8 \\
\hline & & & & HG7LG36-HG7LG38 & 8 \\
\hline \multirow[t]{2}{*}{8} & 4 & 18 & $18 \%$ & HG8LG41-HG8LG23 & 5 \\
\hline & & & & HG8LG92-HG8LG93 & 5 \\
\hline
\end{tabular}


Table 5 The difference ratio of genetic distance in the common marker intervals within the sugarcane HGs between the selected LG A and the other LGs within the same HG B

\begin{tabular}{|c|c|c|c|c|c|c|c|}
\hline HG & LG & No. of shared marker intervals & $\mathrm{LG}_{\max }(\mathrm{cM})$ & $\mathrm{LG}_{i}(\mathrm{cM})$ & $\sum\left|L G_{m a x}-L G_{i k}\right|(\mathrm{cM})$ & $\left(\mathrm{LG}_{\max }+\mathrm{LG}_{i}\right)(\mathrm{cM})$ & Difference ratio \\
\hline \multicolumn{8}{|l|}{ HG1 } \\
\hline LG8 & LG24 & 10 & 747.7 & 310.6 & 437.1 & 1058.4 & 0.4 \\
\hline LG8 & LG77 & 10 & 463.7 & 233.6 & 230.1 & 697.3 & 0.3 \\
\hline LG8 & LG25 & 6 & 38.3 & 63.5 & 25.2 & 101.9 & 0.3 \\
\hline LG8 & LG6 & 10 & 262.3 & 248.6 & 13.7 & 510.9 & 0.0 \\
\hline LG8 & LG75 & 3 & 173.3 & 96.3 & 76.9 & 269.6 & 0.3 \\
\hline LG8 & LG97 & 6 & 81.7 & 60.2 & 21.5 & 141.9 & 0.2 \\
\hline \multicolumn{8}{|l|}{ HG2 } \\
\hline LG1a & LG1b & 15 & 1776.3 & 360.0 & 1416.3 & 2136.3 & 0.7 \\
\hline LG1a & LG1C & 10 & 96.4 & 25.2 & 71.2 & 121.6 & 0.6 \\
\hline LG1a & LG27 & 6 & 58.4 & 83.2 & 24.8 & 141.6 & 0.2 \\
\hline LG1a & LG35 & 6 & 268.0 & 136.7 & 131.3 & 404.7 & 0.3 \\
\hline \multicolumn{8}{|l|}{ HG3 } \\
\hline LG1 & LG55 & 1 & 37.1 & 29.5 & 7.6 & 66.6 & 0.1 \\
\hline LG1 & LG10 & 3 & 170.4 & 84.2 & 86.2 & 254.6 & 0.3 \\
\hline LG1 & LG128 & 1 & 1.0 & 1.1 & 0.1 & 2.1 & 0.1 \\
\hline LG1 & LG112 & 1 & 21.3 & 24.1 & 2.8 & 45.4 & 0.1 \\
\hline \multicolumn{8}{|l|}{ HG4 } \\
\hline LG1a & LG3 & 6 & 434.7 & 278.7 & 155.9 & 713.4 & 0.2 \\
\hline LG1a & LG73 & 6 & 6.3 & 35.7 & 29.3 & 42.0 & 0.7 \\
\hline LG1a & LG131 & 1 & 7.6 & 4.7 & 2.8 & 12.3 & 0.2 \\
\hline LG1a & LG74 & 3 & 7.2 & 3.5 & 3.7 & 10.7 & 0.3 \\
\hline LG1a & LG1C & 1 & 0.9 & 1.8 & 0.8 & 2.7 & 0.3 \\
\hline \multicolumn{8}{|l|}{ HG5 } \\
\hline LG46 & LG47 & 3 & 11.3 & 2.4 & 8.9 & 13.7 & 0.6 \\
\hline LG46 & LG84 & 1 & 8.9 & 29.4 & 20.5 & 38.2 & 0.5 \\
\hline LG46 & LG52 & 1 & 12.7 & 14.1 & 1.5 & 26.8 & 0.1 \\
\hline \multicolumn{8}{|l|}{ HG6 } \\
\hline LG20 & LG140 & 1 & 6.3 & 0.5 & 5.9 & 6.8 & 0.9 \\
\hline LG20 & LG104 & 1 & 5.3 & 0.5 & 4.9 & 5.8 & 0.8 \\
\hline LG20 & LG144 & 1 & 7.7 & 0.5 & 7.3 & 8.2 & 0.9 \\
\hline \multicolumn{8}{|l|}{ HG7 } \\
\hline LG40 & LG87 & 1 & 12.1 & 4.8 & 7.3 & 16.9 & 0.4 \\
\hline \multicolumn{8}{|l|}{ HG8 } \\
\hline LG41 & LG61 & 1 & 27.7 & 36.4 & 8.7 & 64.1 & 0.1 \\
\hline LG41 & LG93 & 1 & 17.1 & 24.6 & 7.5 & 41.7 & 0.2 \\
\hline
\end{tabular}

HG3

HG3 contained 218 markers with a total length of $1243.6 \mathrm{cM}$ and an average distance of $5.7 \mathrm{cM}$ between markers. This HG contained 20 LGs which were aligned with 17 multi-allelic SSRs and SNP markers (Figure 2C, Table 3). The overall order of markers was largely consistent between LGs.
HG4

HG4 contained a total of 308 markers covering $1217.2 \mathrm{cM}$ which gave an average distance of 3.9 cM between markers (Figure 2D, Table 3). This HG contained 18 LGs assigned to the HG group using 19 multi-allelic markers with three densely mapped LGs and a number of smaller LGs. Marker order was maintained across the majority 
of linkage groups although some changes were seen. For example msc274 on HG4LG73 was in a different position relative to HG4LG1.

\section{HG5}

HG5 contained 222 markers that covered a distance of $846.6 \mathrm{cM}$ with an average distance of $3.8 \mathrm{cM}$ between markers (Figure 2E, Table 3). This group contained 13 LGs assigned to the HG with 8 multi-allelic markers. The LGs ranged from $225.8 \mathrm{cM}$ with 66 markers to $5.1 \mathrm{cM}$ with 2 markers.

\section{HG6}

HG6 contained 232 markers on 18 LGs which covered $678.3 \mathrm{cM}$ with an average distance between markers of 2.9 cM (Table 3). This HG contained 7 multi-allelic markers and in general the marker order was consistent between LGs. HG6LG20 contained 6 markers at the end of the linkage group that also aligned to HG3. This appears to be a translocation between the two HGs. There is a $10 \mathrm{cM}$ gap between the groups of markers but the groups were formed at a LOD of 20 giving strong evidence that the linkage is real (Figure 2F).

\section{HG7}

HG7 contained 204 markers which covered 1020.5 cM with an average distance between markers of $5 \mathrm{cM}$. This HG consisted of 16 LGs. Again there were fewer multiallelic markers but from the 12 that were present in the group the marker order was consistent between LGs (Figure 2G).

\section{HG8}

HG8 contained 295 loci which covered 1319.2 cM giving an average of $4.4 \mathrm{cM}$ between markers. This homology group contained 23 LGs formed using 9 multi-allelic markers. This HG contained a larger number of low coverage LGs but in general marker order was consistent between LGs (Figure 2H, Table 3).

\section{Comparison to the R570 genetic linkage map}

Using common SSR markers, this current Q165 map can be partially aligned to the R570 genetic map [16]. For most of the HGs in the Q165 map each HG aligns to a single HG in the R570 map [16,17] (Table 6). The major difference was that HG5 in the Q165 genetic map was missing in the R570 genetic map. There were only 3 SSRs in common between the Q165 HG5 and the R570 map and in all 3 cases, alleles from these SSRs mapped to two HGs. The SSRs markers Msscir47 and Msscir54 mapped to both HG5 and 8 in the Q165 map and to HG VI in R570; the latter aligns to HG8 in the Q165 map. The third marker (Msscir34) mapped to HG5 and 3 in the Q165 map and mapped to HGI in the R570 map; HG1 aligns to HG4 in the Q165 map. These incongruous markers could be explained by the mapping of duplicate loci in the two different cultivars which are polymorphic at different loci. Multi allelic markers that map to more than one locus can create problems for the assignment of LGs to HGs in sugarcane, but it is also possible that in a complex polyploid like sugarcane there are differences in chromosome composition between cultivars. Multilocus markers are common in sugarcane; in the present study 22\% of the SSRs mapped in Q165 mapped to two different loci. This phenomenon has also been observed in diploids [25].

\section{Discussion}

\section{Sugarcane DArT markers}

This is the first sugarcane genetic map published which contains DArT markers and our results demonstrate that sugarcane DArT markers are of high quality and can be used to substantially enhance an existing genetic linkage map. The DArT markers generated here integrated into the existing linkage map, demonstrating that they behave in a Mendelian manner. There was a higher level of redundancy observed for the DArT markers than for any of the other marker types but the DArT array used in this study was not filtered for redundancy. In total, $50 \%$ of the

Table 6 Alignment of Q165 HGs with R570 HGs [14,16] using the SSR markers in common

\begin{tabular}{lllll}
\hline HG Q165 & HG R570 [13,14] & Number of SSR markers in common & $\begin{array}{l}\text { Number of SSR markers used } \\
\text { to align the genetic maps }\end{array}$ & Number of incongruous markers \\
\hline HG1 & VII & 5 & $5(20)^{1}$ & $0^{2}$ \\
HG2 & VIII & 7 & $7(27)$ & 0 \\
HG3 & II & 8 & $7(14)$ & $3(6)$ \\
HG4 & I & 5 & $4(7)$ & $1(4)$ \\
HG5 & - & - & - & - \\
HG6 & IV & 1 & $1(1)$ & $1(3)$ \\
HG7 & III & 4 & $3(8)$ & $3(5)$ \\
HG8 & $V I$ & 7 & $6(16)$ & 0 \\
\hline
\end{tabular}

\footnotetext{
${ }^{1}$ Total number of alleles in brackets which were mapped from the SSRs in common
}

${ }^{2}$ The number of SSRs and alleles in square brackets that map to different HGs. 
DArT markers were clustered within $10 \mathrm{cM}$ regions across the genetic map but the location of these clusters corresponded with the location of clusters of other types of markers. This has been seen in previous DArT genetic maps for sorghum and oat $[26,27]$. These high density clusters could correspond to the centromeric regions of chromosomes where large physical distances correspond to small genetic distances, a feature that has been seen before in sorghum [28]. In sorghum, the closest diploid relative of sugarcane, the pericentromeric heterochromatic regions of the chromosomes have been shown to have much lower rates of recombination $(\sim 8.7 \mathrm{Mbp} / \mathrm{cM})$ compared to euchromatic regions $(\sim 0.25 \mathrm{Mbp} / \mathrm{cM})$ [29]. It is also possible that some clusters represent additional regions where chromosomal rearrangements have occurred or where there is introgression from S. spontaneum causing a higher frequency of polymorphism and hence mapped markers.

\section{Q165 genetic map}

This genetic map is a substantial improvement on previously published maps with more than twice as many markers compared to any other sugarcane genetic map. The high number of markers of known sequence contributed by the DArT, EST-SSR, and SNPs, allowed the majority of the LGs (92\%) to be condensed into eight HGs that align to the basic chromosome number of $S$. spontaneum [3,4]. The 160 LGs had a cumulative map length of $9792.7 \mathrm{cM}$ and an average marker density of $4.3 \mathrm{cM}$. This current linkage map increased the total map length by $734.4 \mathrm{cM}$ and decreased the average marker density from $8.43 \mathrm{cM}$ in the previous Q165 map [13] to $4.3 \mathrm{cM}$. It is known from cytogenetic analysis that Q165 has 110 chromosomes [5]. In this study, the number of LGs greatly exceeds this number of chromosomes although 57 of the LGs are small with 3 or fewer markers (34\%). This result gives an indication of the uneven coverage of markers across the genetic map which has been seen in all of the current published maps, whether generated from selfed populations $[16,19]$ or bi-parental crosses $[17,18,20]$. Sugarcane cultivars have over 100 chromosomes and are all derived from an initial hybrid between the two different species, S. spontaneum and $S$. officinarum followed by backcrossing to the high sugar S. officinarum or other cultivars. As a result, the background S. officinarum part of the genome has lower levels of polymorphism than the S. spontaneum part of the genome. In addition, it is well documented that $2 n$ transmission of chromosomes occurs in interspecific crosses of sugarcane [30]. The introgressed S. spontaneum parts of the genome are the polymorphic regions and in part are responsible for the high density marker areas of the map. The high polyploidy of sugarcane means only a proportion of the markers that are polymorphic can be mapped. Any regions or marker alleles present at greater than two doses can not be accurately mapped with the present population size. The resulting Q165 map has the largest number of markers of any sugarcane linkage map published to date, with 2267 markers mapped across the genome, more than doubling the previous number of markers. However, this still only generates an average of 21 markers per chromosome.

\section{Marker order}

Marker order was in good agreement between LGs within HG1, 4, 6 and 7 (Figure 2). Inconsistencies were observed in groups of markers that were closely linked. Inversion is a common feature of closely spaced markers [25] and could be real, or due to error, or could be explained by the statistical uncertainty of orders at the cM-scale that is inherent in large data sets. In contrast, marker order in HG2 and 8 was less consistent and could be explained by the fact these two groups contain translocated chromosomes. HG3 and 5 also appear to have simple translocations with marker order mostly maintained between LGs within a HG. Translocations have been identified in other polyploids; including wheat, an allopolyploid which has the well documented translocations between 4A, 5A and 7B [31,32].

\section{Chromosome pairing}

In total, repulsion was detected between 30 (19\%) linkage groups, similar to the level detected in R570 where 18 LGs (21\%) were involved in preferential pairing [17]. Analysis of all pairing frequencies determined that complete disomic behaviour was not present, rather the LGs preferentially paired and often within groups of 3 or 4 LGs. A cytogenetic study of Q165 demonstrated that its chromosomal make-up is 15-17 (15\%) S. spontaneum chromosomes, 11-12 (11\%) recombinant chromosomes [5] and 82-83 (75\%) S. officinarum chromosomes. In total, Q165 contains 26-29 chromosomes with complete or partial $S$. spontaneum origin which is a similar number to the 31 LG identified with preferential pairing in this study. It is possible that the LGs displaying preferential pairing are the ones that are inherited from S. spontaneum. To determine how similar LGs were within a homology group, a difference ratio in genetic distance was calculated between LGs within a HG where marker intervals were shared $[24,25,33]$. The number of shared intervals varied between HGs and the accuracy of the difference ratio is probably higher in HG1-4 due to the larger numbers of shared intervals (Table 5). Combining this information with the preferential pairing results (number of LGs in repulsion) showed that HG3 had an average difference ratio of 0.13 and no preferential pairing, whilst HG6 had a ratio of 0.86 with 7 LGs involved in pairing. There was a positive correlation between the difference ratio and the percent 
of LGs detected in repulsion within a HG (Additional file 2) with an $R^{2}=0.44$. The results indicate that within a HG group, as expected, there are two sets of LGs, each likely to be inherited from a different species. Furthermore, the HGs where these two sets of chromosomes are more distinct, for example HG6, showed more preferential pairing within and not between the two sets of LGs. In contrast, for HG3 where the LGs are more similar to each other, there is no preferential pairing.

\section{Comparison to R570}

The majority of the Q165 HGs could be aligned with the R570 HGs using a total of 111 allelic makers from 37 SSRs (Table 6). The Q165 map forms 8 HGs aligning to the basic chromosome number of S. spontaneum. In the case of HGs 2 and 8 for Q165 and VIII and VI in R570 there appear to be more LGs than in the other HGs [17] Table 6. This could be because two sets of S. officinarum chromosomes are aligned with one set of $S$. spontaneum chromosomes which are the result of simple fusion [17]. This was first indicated in the R570 RFLP map [34] and verified in the more recent genetic map [17]. The R570 map is formed into $7 \mathrm{HGs}$ and when aligned to the Q165 map appears to be missing HG5. HG5 in the Q165 map has 8 multi-allelic SSR and SNP markers and an additional 7 single SNP markers. From the location of the SSR markers Smc1047 and SM1420, HG5 appears to contain a translocation from HG2. It is possible that the R570 map lacks this HG5 because these duplicated SSRs have lead to the mis-assembly of sets of homologous LGs into the same HG [17]. It is likely that there will be complex structural differences between the basic chromosome sets of S. officinarum and S. spontaneum which may have lead to misassembly of HGs in both the R570 and Q165 maps. In the Q165 map there appears to be at least 3 LGs (HG5LG47, HG5LG46, HG5LG84) with this same translocation providing strong evidence for its existence (Figure 2).

\section{Conclusions}

We have demonstrated that sugarcane DArT markers provide high quality markers that can be used to construct SD genetic maps in polyploid sugarcane. The high numbers of DArT markers generated in a single assay which are distributed over the whole genome offers a real advantage for a range of molecular breeding and genomics applications. As sugarcane cultivars have approximately 100-120 chromosomes, large numbers of markers are needed to generate a useful genetic map. The highly parallel and automated platforms used in DArT generation mean that the cost per data point is very low. The 2267 markers in this genetic map allowed 92\% of the LGs to be placed into 8 HGs conforming to the basic chromosome number of the ancestral species, S. spontaneum. The large number of SSR markers on this genetic map provides the opportunity for the first time to compare genetic maps derived from different sugarcane cultivars. The cross-compatible markers used to generate the map provide the basis for further studies of comparative structural genomics within cultivated sugarcane varieties and within the Saccharum genus. The use of DArT markers will allow the development of consensus genetic maps in sugarcane which would improve genome coverage and allow integration with other genomic resources. A high coverage genetic map is essential for the correct assembly of the sugarcane genome sequence which a number of research groups are generating.

\section{Methods}

Plant material

The mapping population consisted of 227 progeny derived from a cross between a $S$. officinarum clone IJ76-514 $(2 n=80)$ as the female parent and Q165 $(2 n=110)$, an old Australian cultivar and elite parent.

\section{Marker generation}

The marker data consisted of the original data from [15] to which were added over twice as many new markers generated with AFLP, SSR, RFLP, SNP and DArT markers. Generation of further SSR, and Pst I/MseI AFLP marker data were as reported in the previous Q165 genetic map [15]. All SSR markers are named using the same convention as in [15]. An additional 43 SSR markers were added which were generated from sorghum (with prefix Txp) $[35,36]$. The DArT data was generated as previously described [22]. To increase the number of polymorphic markers, the population was screened twice more across the sugarcane DArT array after cutting the DNA with an extra enzyme, BstN I or Hae III. The DArT markers were designed with the clone number followed by PTB for the BstNI digestion, PTH for the HaeIII digestion and PTP for the original Pst I digestion. DArT markers with a quality score of $\mathrm{Q}>80 \%$ and a call rate of at least $80 \%$ were selected for mapping. The SNP data was generated using a Sequenom assay developed as previously described in [37]. The SNP markers were named with their gene index name and an allele number. Gene Index names correspond to the Saccharum officinarum Gene Indices at the Computational Biology and Functional Genomics laboratory at the Dana-Farber Cancer Institute (http://compbio.dfci.harvard.edu/tgi/cgi-bin/ tgi/gimain.pl?gudb = s_officinarum). The 27 RFLP markers were generated as previously described in [38], and they were named with a clone number from a sugarcane cDNA library and the enzyme used for digestion. An additional two markers were derived from maize genomic cDNA prefix UMC [39]. 


\section{Genetic mapping}

All marker systems produced monomorphic and polymorphic fragments. Each segregating band was scored independently as a dominant marker (presence vs. absence). As sugarcane is highly polyploid only SD markers were used for map construction, that is, markers that were present in Q165 and absent in IJ76-514. These markers displayed segregation ratios that did not significantly differ from $1: 1$ [7] at $\mathrm{P}=0.05$ by the $\mathrm{X}^{2}$ test [13] The newly generated SD markers were added to the 967 existing markers from [15]. The new map was constructed using the software Joinmap 4.0 [40]. Marker groups were formed by two-point analysis at a LOD score threshold of at least 10 and a recombination fraction of 0.35 . The linkage groups (LG) were then ordered using standard methods in JoinMap. The maps were generated in two steps. Initially only markers that were run on at least 188 progeny were used to generate a framework map, and then additional DArT markers that were only scored on 94 progeny were added to the framework map. LGs were then assembled into homology groups (HGs) using a minimum of two common markers.

\section{Investigation of chromosome pairing}

Chromosome pairing was investigated by analysing markers in repulsion. The segregation data matrix was inverted then combined with the original data. A two point analysis was then carried out to identify linkage between the original data and the reverse phase data to identify pairs of markers in repulsion at a $L O D \leq 5$. The distribution of these markers was analysed, and repulsion only accepted where large parts of the LG were involved, as artefactual repulsion is more likely to involve single pairs of markers $[7,41]$. To determine how variation between the LGs within a HG affects chromosome pairing, a distance measurement of intervals between two individuals [24] as modified by [33], was used to compare the genetic distances between LGs within a HG to the most densely mapped LG within the same HG. The modified distance measure:

$$
\text { Difference ratio }=\sum \mid \frac{\mathrm{LG}_{\max }-\mathrm{Lg}_{i k}}{\mathrm{LG}_{\max }+\mathrm{Lg}_{i}}
$$

Where $\mathrm{LG}_{\max }$ is the length (cM) of the $k$ th shared marker interval on the most densely mapped LG within a HG and $\mathrm{Lg}_{\text {ik }}$ is the length (cM) of the $k$ th shared marker interval on the $j$ th LG within the same HG. The $\sum\left|L_{G_{m a x}}-L_{j k}\right|$ is the absolute value of the length difference of each shared marker interval within a HG between $L G$ s $L_{\text {max }}$ and $L G_{i}$ and $L_{\text {max }}+L_{i}$ is an additive value of all shared intervals for the $i$ th $L G$ within $\mathrm{HG}$ which is used to normalise the difference value $\sum\left|\mathrm{LG}_{\max }-\mathrm{LG}_{\mathrm{jk}}\right|$ [33]. These values were then averaged within a HG to give an average difference ratio.

\section{Additional files}

Additional file 1: Q165 genetic map. Excel spreadsheet containing a list of all map loci, and map positions in each HG.

Additional file 2: A graph of the correlation between number of LGs within a HG in repulsion and the average distance ratio calculated from Table 5 .

\section{Competing interests}

The authors declare that they have no competing interests.

\section{Authors' contributions}

KSA carried out the mapping analysis and drafted the manuscript. AK and KUH performed the DArT data production. SH, JL and MDM generated the AFLP and SSR data, PCJ and RJH contributed the SNP data. KA, MDM, PCJ and $\mathrm{RJH}$ critically revised the manuscript. All authors read and approved the final manuscript.

\section{Acknowledgements}

The authors are grateful for funding contributions from the following organisations: Australian Sugar Research and Development Corporation; Cooperative Research Centre for Sugar Industry Innovation through Biotechnology; CSIRO and BSES Limited. We thank A. Rae, G. Bonnett and L. C. Mclntyre for critical reading of the manuscript.

\section{Author details}

'CSIRO Plant Industry, Queensland Bioscience Precinct, 306 Carmody Rd, St Lucia, QLD 4067, Australia. ²BSES Limited, Meiers Road, Indooroopilly, QLD 4068, Australia. ${ }^{3}$ Southern Cross Plant Science, Southern Cross University, Military Rd, Lismore, NSW 2480, Australia. ${ }^{4}$ Diversity Arrays, Technology Pty Ltd, PO Box 7141, Yarralumla, ACT 2600, Australia. ${ }^{5}$ Queensland Alliance for Agriculture and Food Innovation, University of Queensland, Brisbane, QLD 4072, Australia.

Received: 5 September 2013 Accepted: 6 February 2014 Published: 24 February 2014

\section{References}

1. Carruthers SP: Solid biofuels: fuel crops. In Crops for Industry and Energy 1994 CAS Report 15:168-180. Edited by Carruthers SP, Miller PA, Vaughan CMA. Centre for Agricultural Strategy, University of Reading UK; 1994.

2. Matsuoka S, Ferro J, Arruda P: The Brazilian experience of sugarcane ethanol industry. In Biofuels - Global Impact on Renewable Energy, Production Agriculture and Technological Advancements. Edited by Tomes D, Lakshmanan P, Songstad D. New York, Dordrecht, Heidelburg, London: Springer; 2011:157-172

3. D'Hont A, Ison D, Alix K, Roux C, Glaszmann JC: Determination of basic chromosome numbers in the genus Saccharum by physical mapping of ribosomal RNA genes. Genome 1998, 41:221-225.

4. D'Hont A, Grivet L, Feldmann P, Rao PS, Berding N, Glauszmann JC: Characterisation of the double genome structure of modern sugarcane cultivars (Saccharum spp.) by molecular cytogenetics. Mol Gen Genet 1996, 250:404-413.

5. Piperidis G, Piperidis N, D'Hont A: Molecular cytogenetic investigation of chromosome composition and transmission in sugarcane. Mol Gen Genet 2010, 284:65-73.

6. Le Cunff L, Garsmeur O, Raboin LM, Pauquet J, Telismart H, Selvi A, Grivet L, Philippe R, Begum D, Deu M, Costet L, Wing R, Glaszmann JC, D'Hont A: Diploid/polyploid syntenic shuttle mapping and haplotype-specific chromosome walking towards a rust resistance gene (Brul) in highly polyploid sugarcane (2n 12x 115). Genetics 2008, 180:649-660.

7. Wu KK, Burnquist W, Sorrels ME, Tew TL, Moore PH, Tanksley SD: The detection and estimation of linkage in polyploids using single-dose restriction fragments. Theor Appl Genet 1992, 83:294-300. 
8. Grattapaglia D, Sederoff R: Genetic linkage maps of eucalyptus grandis and eucalyptus urophylla using pseudo-testcross: mapping strategy and RAPD markers. Genetics 1994, 137:1121-1137.

9. Al-Janabi SM, Honeycutt RJ, McClelland M, Sobral BWS: A genetic linkage map of Saccharum spontaneum L. 'SES208'. Genetics 1993, 134:1249-1260.

10. Da Silva J, Honeycutt RJ, Burnquist W, Al—Janabi SM, Sorrells ME, Tanksley SD, Sobral BWS: Saccharum spontaneum L. 'SES208' genetic linkage map combining RFLP- and PCR-based markers. Mol Breed 1995, 1:165-179.

11. Ming R, Liu S-C, Lin Y-R, da Silva J, Wilson W, Braga D, van Deynze A, Wenslaff TF, Wu KK, Moore PH, Burnquist W, Sorrells ME, Irvine JE, Paterson $\mathrm{AH}$ : Detailed alignment of Saccharum and Sorghum Chromosomes: Comparative organisation of closely related diploid and polyploidy genomes. Genetics 1998, 150:1663-1682.

12. Mudge J, Andersen WR, Kehrer RL, Fairbanks DJ: A RAPD genetic map of Saccharum officinarum. Crop Sci 1996, 36:1362-1366.

13. Aitken KS, Jackson PA, McIntyre CL: Construction of a genetic linkage map for Saccharum officinarum incorporating both simplex and duplex markers to increase genome coverage. Genome 2007, 50:742-756.

14. Hoarau JY, Offmann B, D'Hont A, Risterucci AM, Roques D, Glaszmann JC, Grivet L: Genetic dissection of a modern sugarcane cultivar (Saccharum spp.). 1. Genome mapping with AFLP markers. Theor App/ Genet 2001, 103:84-97.

15. Aitken $\mathrm{KS}$, Jackson PA, Mclntyre CL: A combination of AFLP and SSR markers provides extensive map coverage and identification of homo(eo)logous linkage groups in a sugarcane cultivar. Theor App/ Genet 2005, 110:789-801.

16. Rossi M, Araujo PG, Paulet F, Garsmeur O, Dias VM, Chen H, Van Sluys MA, D'Hont A: Genomic distribution and characterisation of EST-derived resistance gene analogs (RGAs) in sugarcane. Mol Gen Genomics 2003, 269:406-419.

17. Raboin LM, Oliveira KM, Lecunff L, Telismart $H$, Roques D, Butterfield M, Hoarau JY, D'Hont A: Genetic mapping in sugarcane, a high polyploidy, using bi-parental progeny: identification of a gene controlling stalk colour and a new rust resistance gene. Theor App/ Genet 2006, 113:1382-1391.

18. Oliveira KM, Pinto LR, Marconi TG, Margarido GRA, Pastina MM, Teixeira LHM, Figueira AV, Ulian EC, Garcia AAF, Souza AP: Functional integrated genetic linkage map based on EST-markers for a sugarcane (Saccharum spp.) commercial cross. Mol Breed 2007, 20:189-208.

19. Andru S, Pan YB, Thongthawee S, Burner DM, Kimbeng CA: Genetic analysis of the sugarcane (Saccharum spp.) cultivar 'LCP 85-384'. 1. Linkage mapping using AFLP, SSR and TRAP markers. Theor App/ Genet 2011, 123:77-93.

20. Palhares AC, Rodrigues-Morais TB, Van Sluys MA, Domingues DS, Maccheroni W, Jordão H, Souza AP, Marconi TG, Mollinari M, Gazaffi R, Garcia AAF, Carneiro Vieira ML: A novel linkage map of sugarcane with evidence for clustering of retrotransposon-based markers. BMC Genet 2012, 13:51.

21. Wenzl P, Carling J, Kudrna D, Jaccoud D, Huttner E, Kleinhofs A, Kilian A: Diversity arrays technology (DArT) for whole-genome profiling of barley. Proc Natl Acad Sci U S A 2004, 101:9915-9920.

22. Heller-Uszynska K, Uszynski G, Huttner E, Evers M, Carlig J, Caig V, Aitken K, Jackson P, Piperidis G, Cox M, Gilmour R, D'Hont A, Buterfield M, Glaszmann JC, Kilian A: Diversity arrays technology effectively reveals DNA polymorphism in a large and complex genome of sugarcane. Mol Breed 2010, 28:37-55.

23. Baker $\mathrm{P}$, Jackson PA, Aitken K: Bayesian estimation of marker dosage in sugarcane and other autopolyploids. Theor App/ Genet 2010, 120:1653-1672.

24. Gower JC: A general coefficient of similarity and some of its properties. Biometrics 1971, 27:857-874.

25. Mace ES, Rami J-F, Bouchet S, Klein PE, Klein RR, Kilian A, Wendzl P, Xia L, Halloran $K$, Jordan D: A consensus genetic map of sorghum that integrates multiple component maps and high-throughput Diversity Array Technology (DArT) markers. BMC Plant Biol 2009, 9:1-14.

26. Mace ES, Xia L, Jordan DR, Halloran K, Parth DK, Huttner E, Wenzl P, Kilian A: DArT markers: diversity analyses and mapping in Sorghum bicolour. BMC Genomics 2008, 9:26-37.

27. Tinker NA, Kilian A, Wight CP, Heller-Uszynska K, Wenzl P, Rines HW, Bjørnstad, Howarth CJ, Jannink J-L, Anderson JM, Rossnagel BG, Stuthman DD, Sorrells ME, Jackson EW, Tuvesson S, Kolb FL, Olssom O, Federizzi LC, Carson ML, Ohm HW, Molnar SJ, Scoles GJ, Eckstein PE, Bonman JM, Ceeplitis A, Langdon T: New DArT markers for oat provide enhanced map coverage and global germplasm characterisation. BMC Genomics 2009, 10:39-61.

28. Bowers JE, Abbey C, Anderson S, Chang C, Draye X, Hoppe AH, Jessup R, Lemke C, Lennington J, Li Z, Lin Y, Liu S, Luo L, Marler BS, Ming R, Mitchell SE, Qiang D, Reischmann K, Schulze SR, Skinner DN, Wang Y, Kresovich S,
Schertz KF, Paterson AH: A high-density genetic recombination map of sequence-tagged sites for Sorghum, as a framework for comparative structural and evolutionary genomics of tropical grains and grasses. Genetics 2003, 165:367-386.

29. Kim J-S, Islam-Faridi MN, Klein PE, Stelly DM, Price HJ, Klein RR, Mullet JE: Comprehensive molecular cytogenetic analysis of sorghum genome architecture: distribution of euchromatin, heterochromatin. Genes and recombination in comparison to rice. Genetics 2005, 171:1963-1976.

30. Bhat SR, Gill SS: The implications of $2 n$ egg gametes in mobilization and breeding of sugarcane. Euphytica 1985, 34:377-384.

31. Devos KM, Dubcovsky J, Dvořák J, Chinoy CN, Gale MD: Structural evolution of wheat chromosomes $4 \mathrm{~A}, 5 \mathrm{~A}$ and $7 \mathrm{~B}$ and its impact on recombination. Theor App/ Genet 1995, 91:282-288.

32. Berkman PJ, Skarshewski A, Manoli S, Lorenc MT, Stiller J, Smits L, Lai K, Campbell E, Kubaláková M, Šimková H, Batley J, Doležel, Hernandez P, Edwards D: Sequencing wheat chromsomes arm 7BS delimits the 7BS/ 4AL translocation and reveals homoeologous gene conversion. Theor Appl Genet 2012, 124:423-432.

33. Wu YQ, Huang Y: An SSR genetic map of Sorghum bicolour (I.) Moench and its comparison to a published genetic map. Genome 2007, 50:84-89.

34. Grivet L, D'Hont A, Roques D, Feldmann P, Lanaud C, Glasmann JC: RFLP mapping in a highly polyploidy and aneuploid interspecific hybrid. Genetics 1996, 142:987-1000.

35. Bhattramakki D, Dong J, Chhabra AK, Hart GE: An integrated SSR and RFLP linkage map of Sorghum bicolour (L.) Moench. Genome 2000, 43:988-1002.

36. Kong L, Dong J, Hart GE: Characteristics, linkage map positions and alleleic differentiation of Sorghum bicolour (L.) Moench DNA simple-sequence repeats (SSRs). Theor App/ Genet 2000, 101:438-448.

37. Bundock PC, Eliott FG, Ablett G, Benson AD, Casu RE, Aitken KS, Henry RJ: Targeted single nucleotide polymorphism (SNP) discovery in a highly polyploidy plant species using 454 sequencing. Plant Biotechnol J 2009, 7:347-354

38. Chao S, Sharp PJ, Worland AJ, Warham EJ, Koebner RMD, Gale MD: RFLP-based genetic maps of wheat homologous group-7 chromosomes. Theor App/ Genet 1989, 78:495-504.

39. Tao YZ, Jordan DR, Mclntyre CL, Henzell RG: Construction of a genetic map in a sorghum recombinant inbred line using probes from different sources and its comparison with other sorghum maps. Australian J Agric Res 1998, 49:729-736.

40. Van Ooijen JW: JoinMap ${ }^{\circledR} 4$, Software for the calculation of genetic linkage maps in experimental populations. Wageningen, Netherlands: Kyazma B.V; 2004

41. Qu L, Hancock JF: Detecting and mapping repulsion -phase linkage in polyploids with polysomic inheritance. Theor App/ Genet 2001, 103:136-143.

doi:10.1186/1471-2164-15-152

Cite this article as: Aitken et al: A comprehensive genetic map of sugarcane that provides enhanced map coverage and integrates high-throughput Diversity Array Technology (DArT) markers. BMC Genomics 2014 15:152.

\section{Submit your next manuscript to BioMed Central and take full advantage of:}

- Convenient online submission

- Thorough peer review

- No space constraints or color figure charges

- Immediate publication on acceptance

- Inclusion in PubMed, CAS, Scopus and Google Scholar

- Research which is freely available for redistribution 